\title{
GMR
}

\section{Artesunate modulates expression of matrix metalloproteinases and their inhibitors as well as collagen-IV to attenuate pulmonary fibrosis in rats}

\author{
Y. Wang*, G. Huang*, B. Mo* and C. Wang \\ Division of Respiratory Diseases, \\ Affiliated Hospital of Guilin Medical University, Guilin, Guangxi, China \\ *These authors contributed equally to this study. \\ Corresponding author: C. Wang \\ E-mail: 1507959392@qq.com
}

Genet. Mol. Res. 15 (2): gmr.15027530

Received August 27, 2015

Accepted November 5, 2015

Published June 3, 2016

DOI http://dx.doi.org/10.4238/gmr.15027530

\begin{abstract}
The aim of this study was to determine the effect of artesunate on extracellular matrix (ECM) accumulation and the expression of collagen-IV, matrix metalloproteinase (MMP), and tissue inhibitor of matrix metalloproteinase (TIMP) to understand the pharmacological role of artesunate in pulmonary fibrosis. Eighty Sprague-Dawley rats were randomly assigned to four groups that were administered saline alone, bleomycin (BLM) alone, BLM + artesunate, or artesunate alone for 28 days. Lung tissues from 10 rats in each group were used to obtain lung fibroblast (LF) primary cells, and the rest were used to analyze protein expression. The mRNA expression of collagen-IV, MMP-2, MMP-9, TIMP-1, and TIMP-2 in lung fibroblasts was detected by real-time quantitative reverse transcriptase polymerase chain reaction. The protein levels of collagen-IV, MMP-2, MMP-9, TIMP-1, and TIMP-2 protein in lung tissues were analyzed by western blotting. Artesunate treatment alleviated alveolitis and pulmonary
\end{abstract}


fibrosis induced by bleomycin in rats, as indicated by a decreased lung coefficient and improvement of lung tissue morphology. Artesunate treatment also led to decreased collagen-IV protein levels, which might be a result of its downregulated expression and increased MMP-2 and MMP-9 protein and mRNA levels. Increased TIMP-1 and TIMP2 protein and mRNA levels were detected after artesunate treatment in lung tissues and primary lung fibroblast cells and may contribute to enhanced activity of MMP-2 and -9. These findings suggested that artesunate attenuates alveolitis and pulmonary fibrosis by regulating expression of collagen-IV, TIMP-1 and 2, as well as MMP-2 and -9, to reduce ECM accumulation.

Key words: Pulmonary fibrosis; Artesunate; Matrix metalloproteinases; Tissue inhibitor of matrix metalloproteinase; Extracellular matrix; Collagen-IV

\section{INTRODUCTION}

Pulmonary fibrosis is a common chronic lung disease with high mortality (Leppäranta et al., 2012). Presently, there are no effective treatments for this disorder. Its typical features include epithelial injury, fibroblastic proliferation, inflammation, and increased matrix production (du Bois, 2010). During the pathogenetic process, a large amount of ECM deposition forms the fibroblast foci, which may play a key role in idiopathic pulmonary fibrosis (IPF). MMPs are a group of Zn-dependent proteinases capable of degrading ECM, which are activated by proteolytic removal of their amino terminal and are inhibited by TIMPs (Dancer et al., 2011). A series of MMPs such as MMP-1, MMP-2, MMP-7, and MMP-9 were upregulated in IPF and in experimental models of pulmonary fibrosis (Checa et al., 2008; García-de-Alba et al., 2010; Pardo and Selman, 2012; Song et al., 2013; Yamashita et al., 2011). MMP-2 and MMP-9 are also called gelatinase A and gelatinase B, respectively, and degrade collagen type IV, the major component of the basement membrane, and induce lung tissue remodeling and progressive alveolar thickening, causing the alveolar cavity to shrink and even disappear, leading to pulmonary fibrosis. MMP-2 is usually upregulated in experimental models of lung fibrosis, and the overexpression of MMP-2 and 9 have been proposed to play a key role in basement membrane disruption (Van Doren, 2015). Because the structural integrity of the alveolar wall depends on the basement membrane, destruction of the subepithelial basement membrane may be an early event during the process of alveolar fibrosis. TIMP-1 is a 28.5 $\mathrm{kDa}$ glycoprotein that forms a complex with activated MMPs as well as with the proenzyme form of MMP-9 (Akkoc and Kahraman, 2012; Kiani et al., 2013; Wang et al., 2015b). TIMP2 is a $21 \mathrm{kDa}$ protein that shares $37 \%$ amino acid identity and $65.6 \%$ overall homology with TIMP-1. However, TIMP-1 and -2 are immunologically distinct (Song et al., 2013). In IPF, the myofibroblasts and the epithelial cells of the buds showed moderate to strong reactions to TIMP-1 and TIMP-2 antibodies (Kisarova and Korolenko, 2012).

Although significant progress has been made in understanding the molecular mechanisms of the pathogenesis of pulmonary fibrosis, there are no effective therapies for this disease. Thus, it is necessary to identify new drugs with satisfactory efficacy for pulmonary fibrosis. Artesunate is a semi-synthetic derivative of artemisinin and an effective medicine for 
malaria. Recently, we demonstrated that artesunate has anti-profibrotic activity in rats with bleomycin-induced pulmonary fibrosis (Li et al., 2014). However, the underlying mechanisms remain unclear. This study investigated the effects of artesunate on the expression of collagenIV, MMP-2, MMP-9, TIMP-1, and TIMP-2 in the primary lung fibroblasts and the lung tissues from pulmonary fibrosis rats treated with artesunate.

\section{MATERIAL AND METHODS}

\section{Materials}

Bleomycin was purchased from Zhejiang Hisun Pharmaceutical Co., Ltd. (Taizhou, China). Artesunate was purchased from Guilin Pharmaceutical Co., Ltd. (Guilin, China). Primers were synthesized by Invitrogen Life Technologies (Shanghai, China). The cDNA synthesis kit was purchased from Takara Biotechnology Co., Ltd. (Dalian, China). The total RNA extraction kit and 2x Taq PCR Master mix were obtained from Tiangen Biotech Co., Ltd. (Beijing, China). The TIMP-1 and TIMP-2 antibodies and horseradish peroxidase-conjugated secondary antibodies were purchased from Beijing Zhongshan Golden Bridge Biotechnology Co., Ltd. (Beijing, China). Collagen-IV antibody was purchased from Abcam Ltd. (Hong Kong, China). MMP-2 antibody and MMP-9 antibodies were purchased from Santa Cruz Biotechnology, Inc. (Hong Kong, China).

\section{Animals and experimental protocols}

Male Sprague Dawley (SD) rats weighing $200 \pm 30$ g (from Animal Resources Center of Guilin Medical University, Guilin, China) were used in this study. The rats were housed in specific pathogen-free conditions with free access to food and water.

Animal protocols were approved by the Guilin Medical University Animal Experiment Ethics Committee. Rats were randomly assigned to each of the following groups: 1) Control group $(\mathrm{N}=20)$, which received intratracheal administration of $0.9 \% \mathrm{NaCl}$ solution alone; 2$)$ BLM-treated group $(\mathrm{N}=20)$, which received intratracheal administration of bleomycin (5 $\mathrm{mg} / \mathrm{kg}) ; 3) \mathrm{BLM}+$ artesunate group $(\mathrm{N}=20)$, which received daily intratracheal administration of bleomycin $(5 \mathrm{mg} / \mathrm{kg})$ and daily intraperitoneal injection of artesunate $(100 \mathrm{mg} / \mathrm{kg}) ; 4)$ artesunate group $(\mathrm{N}=20)$, which received daily intraperitoneal injections of artesunate (100 $\mathrm{mg} / \mathrm{kg}$ ). Bleomycin was injected into the trachea exposed through a midline anterior neck incision using a 24-gauge needle. On the 28th day of bleomycin treatment, rats were sacrificed by decapitation under anesthesia by inhaling chloral hydrate (1 mg/100 g; 20130513; Kelong Chemical Reagent Company, Chengdu, China). Lung tissues from 10 rats were processed for lung fibroblast cell culture and RT-PCR, and the tissues from the rest were processed for hematoxylin and eosin (H\&E) staining and western blotting.

\section{Lung fibroblast culture}

Lung tissues were isolated into several lobes, irrigated once with PBS, cut into $1-\mathrm{mm}^{3}$ pieces, and washed with PBS containing penicillin and streptomycin 4 times. Tissues were washed once with $0.1 \%$ trypsin (Gibco, Grand Island, NY, USA) and then digested in $1 \mathrm{~mL}$ $0.1 \%$ trypsin for $20 \mathrm{~min}$ at $37^{\circ} \mathrm{C}$. The digestion was terminated by adding $1 \mathrm{~mL}$ Dulbecco's 
modified Eagle's medium (DMEM) (basic glucose, GE Healthcare HyClone, Logan, UT, USA) supplemented with 10\% fetal bovine serum (FBS) (Gibco, Grand Island, NY, USA). The digested tissue suspension was filtered with 100 mesh sieve, centrifuged at $1500 \mathrm{rpm}$ for 5 min, resuspended with DMEM with $10 \%$ FBS, centrifuged again at $800 \mathrm{rpm}$ for $5 \mathrm{~min}$, and the supernatant (called part A herein) and sediment(called part B herein) were collected. Part A contained most of the fibroblasts and a few epithelial cells, which were then centrifuged at $1500 \mathrm{rpm}$ for $5 \mathrm{~min}$, resuspended in culture medium, and incubated in a $25-\mathrm{mL}$ flask in a $5 \%$ carbon dioxide atmosphere at $37^{\circ} \mathrm{C}$ for $40 \mathrm{~min}$. Then, the medium was replaced. Part B contained more epithelial cells and a few fibroblasts, which were then centrifuged at $1500 \mathrm{rpm}$ for $5 \mathrm{~min}$, resuspended in culture medium, and incubated in a $25 \mathrm{~mL}$ flask in a $5 \%$ carbon dioxide atmosphere at $37^{\circ} \mathrm{C}$ for $40 \mathrm{~min}$. Then, the medium was replaced. The fibroblasts were purified as follows: after $24 \mathrm{~h}$ of culturing, cells from part A and B were digested with $1 \mathrm{~mL}$ $0.1 \%$ trypsin for $5 \mathrm{~min}$, and the digestion was terminated with $1 \mathrm{~mL} 10 \%$ medium. The cells were centrifuged at $1500 \mathrm{rpm}$ for $5 \mathrm{~min}$ and resuspended in DMEM with $10 \%$ FBS. The purifying process was performed 3 times, and then pure fibroblasts were obtained. Cells at passage 3 were used for further analysis.

\section{RT-PCR analysis}

Total RNA was isolated from the lung tissues using TRIzol reagent (Invitrogen Life

Technologies, Carlsbad, CA, USA) according to the manufacturer instructions. PCR was performed in a $25-\mu \mathrm{L}$ reaction volume, containing the cDNA template $(2 \mu \mathrm{L})$, forward and backward primers $\left(1 \mu \mathrm{L}\right.$ each), $12.5 \mu \mathrm{L} 2 \mathrm{X}$ master mix, and $8.5 \mu \mathrm{LddH}_{2} \mathrm{O}$ for 30 cycles at $94^{\circ} \mathrm{C}$ for $30 \mathrm{~s}, 58^{\circ} \mathrm{C}$ for $30 \mathrm{~s}$, and $72^{\circ} \mathrm{C}$ for $30 \mathrm{~s}$ with a final extension at $72^{\circ} \mathrm{C}$ for $2 \mathrm{~min}$. The primer pairs for the genes encoding collagen-IV, MMP-2, MMP-9, TIMP-1, and TIMP-2 are shown in Table1, and the GAPDH housekeeping gene was used as an internal control.

\begin{tabular}{|c|c|c|c|c|}
\hline \multirow{3}{*}{$\begin{array}{l}\text { Gene } \\
\text { Collagen-IV (COL4A1) }\end{array}$} & \multicolumn{2}{|c|}{ Sequences (5'-3') } & \multirow{2}{*}{$\begin{array}{c}\text { Length (bp) } \\
20\end{array}$} & \multirow{3}{*}{$\begin{array}{c}\text { Applications (bp } \\
86\end{array}$} \\
\hline & Forward: & GTCCTGTCTGGAAGAGTTTA & & \\
\hline & Reverse: & AGCTGTAAGCATTCGCGTAG & 20 & \\
\hline \multirow[t]{2}{*}{$M M P-2$} & Forward: & GGGAATGAGTACTGGGTCTATT & 22 & \multirow[t]{2}{*}{114} \\
\hline & Reverse: & CCAGTTAAAGGCAGCGTCTA & 20 & \\
\hline \multirow[t]{2}{*}{$M M P-9$} & Forward: & GATCAGCCGGGAACGTATCT & 20 & \multirow[t]{2}{*}{172} \\
\hline & Reverse: & AACTACAACGCCAGAAGTAT & 20 & \\
\hline \multirow[t]{2}{*}{ TIMP-1 } & Forward: & GCTTTCTGCAACTCGGACCT & 20 & \multirow[t]{2}{*}{148} \\
\hline & Reverse: & ACCGGAAACCTGTGGCATTT & 20 & \\
\hline \multirow[t]{2}{*}{$T I M P-2$} & Forward: & GCAACAGGCGTTTTGCAATG & 20 & \multirow[t]{2}{*}{93} \\
\hline & Reverse: & GGGTTGCCATAGATGTCAT & 19 & \\
\hline \multirow[t]{2}{*}{ GAPDH } & Forward: & CATCTTCTTGTGCAGTGCCA & 20 & \multirow[t]{2}{*}{132} \\
\hline & Reverse: & GATGGCAACAATGTCCACTT & 20 & \\
\hline
\end{tabular}

\section{Histopathological analysis}

The weight of the rat body and the whole lung tissue were measured to calculate the lung coefficient (Lung coefficient $=$ lung weight/rat body weight $\mathrm{x} 100 \%$ ). For the histological exam, lung tissue was embedded in paraffin, cut into sections, and stained with H\&E. The stained sections were observed under a light microscope, and alveolitis and fibrosis were semi-quantitatively assessed according to the criteria described by Szapiel et al (Szapiel et al., 1979) (Table 2). 
Table 2. Szapiel's grading criteria of alveolitis and pulmonary fibrosis.

\begin{tabular}{l|c|c}
\hline Severity & Alveolitis & Fibrosis \\
\hline None $(0)$ & No & No \\
\hline Mild $(1+)$ & $<20 \%$ of the lung & $<20 \%$ of the lung \\
\hline Moderate $(2++)$ & $20-50 \%$ of the lung & $20-50 \%$ of the lung \\
\hline Severe $(3+++)$ & $>50 \%$ of the lung & $>50 \%$ of the lung \\
\hline
\end{tabular}

\section{Western blotting}

Protein extracts from rat lung tissues were prepared using ice-cold NP-40 lysis buffer (Beyotime, Shanghai, China), and the protein concentration was measured with a bicinchoninic acid protein assay kit (Beyotime, Shanghai, China). The proteins were separated with sodium dodecyl sulfate polyacrylamide gel electrophoresis (SDS-PAGE) and then transferred onto polyvinylidene difluoride membranes (Millipore, Bedford, MA, USA). The membranes were blocked with 5\% (w/v) non-fat milk (Yili Industrial Group Company, Hohhot, China) at $25^{\circ} \mathrm{C}$ for $1 \mathrm{~h}$ and then incubated with primary antibodies (1:500 dilution) at $4^{\circ} \mathrm{C}$ overnight, followed by $3 \times 5$ min washes. The membranes were incubated with secondary antibodies (1:5000 dilution) at $25^{\circ} \mathrm{C}$ for $1 \mathrm{~h}$. The results were visualized using a standard enhanced chemiluminescence (ECL) kit (Millipore), and the band density of interest was measured and normalized using GAPDH.

\section{Statistical analysis}

Statistical analyses were performed with the Sigma plot software (SPSS) version 22.0 software (IBM, Armonk, NY, USA). The data are reported as means \pm standard deviation. Statistical differences were determined by two-way analysis of variance and $\mathrm{P}<0.05$ was considered a significant difference.

\section{RESULTS}

\section{Artesunate attenuates the inflammatory responses in BLM-induced pulmonary fibrosis in rats}

To determine the effect of artesunate on BLM-induced pulmonary inflammation responses in rats, the morphological changes in the lung tissues were examined. The tissues from the BLMtreated group contained many gray nodules, pale in color on the lung surface, giving a rough texture to the surface. In addition, the bullae of the lung were occasionally observed. In the artesunatetreated group, lung fibrosis was alleviated, and hyperemia dropsy and volume augmentation of the lung tissues were observed. As shown in Figure 1, the lung coefficient significantly increased in the BLM-treated group, but it markedly decreased after artesunate treatment.

Compared with those of lung tissues from saline or artesunate groups, the lung structure of the BLM-treated group was severely damaged and the lung interstitium was obviously thickened (Figure 2A). These pathological changes were attenuated by the intraperitoneal injection of artesunate, and were evidenced by the grading of alveolitis and pulmonary fibrosis (Figure 2B and C). Collectively, these results suggested that artesunate protects the lung from the deleterious morphological damages induced by BLM. 


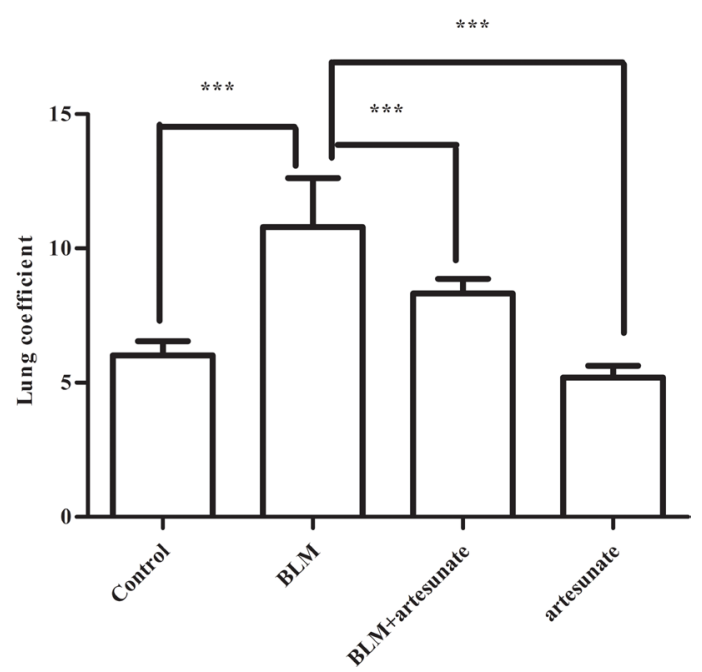

Figure 1. Artesunate inhibited BLM-induced increases in lung coefficient. Results are repored as means \pm standard deviation. ${ }^{*} \mathrm{P}<0.05$ and $* * * \mathrm{P}<0.001$.
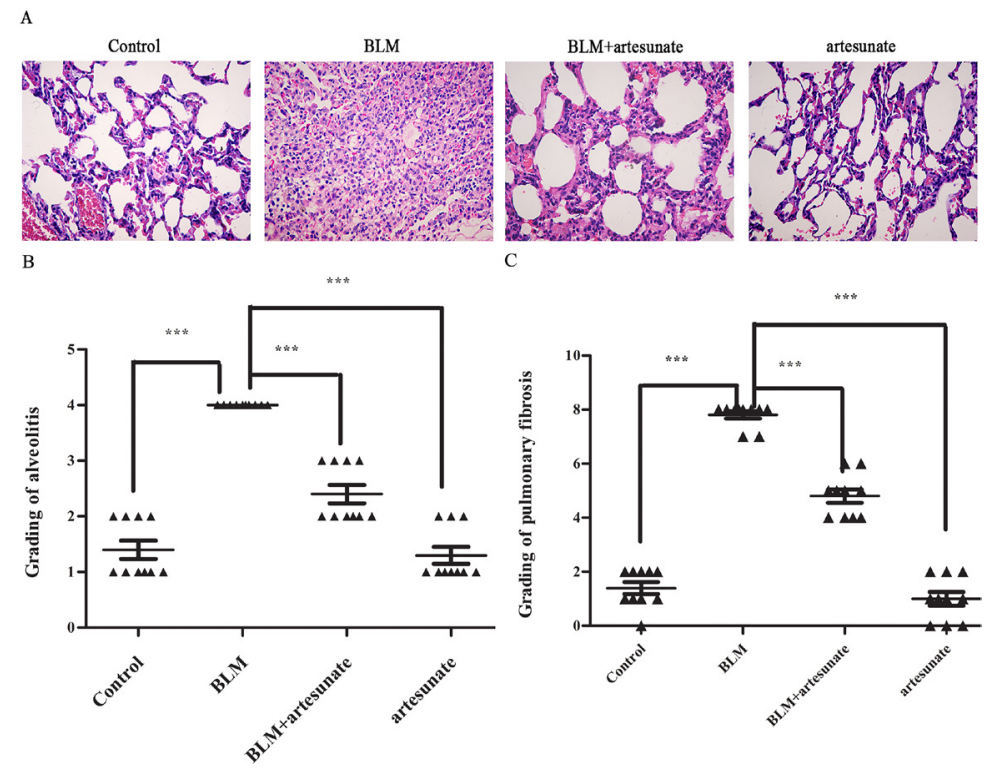

Figure 2. Artesunate dramatically alleviated histological damage in lung tissues. A. Representative images of H\&E staining. B. Grading of alveolitis in lung tissues $(\mathrm{N}=10)$. C. Grading of pulmonary fibrosis in lung tissue $(\mathrm{N}=10)$. Results are reported as means \pm standard deviation. $* \mathrm{P}<0.05$ and $* * * \mathrm{P}<0.001$.

\section{Artesunate inhibits collagen-IV expression in BLM-induced pulmonary fibrosis in rats}

A hallmark of IPF is the abnormal deposition of collagen. Collagen-IV is the major component of alveoli basement membrane. Therefore, we determined collagen-IV protein levels in lung tissues. The data demonstrated that collagen-IV was upregulated in bleomycin- 
treated rats, and artesunate inhibited collagen-IV upregulation induced by bleomycin (Figure $3 \mathrm{~A}$ and $\mathrm{B}$ ), which was consistent with the collagen-IV mRNA levels in lung fibroblasts detected by RT-PCR (Figure 3C and D).

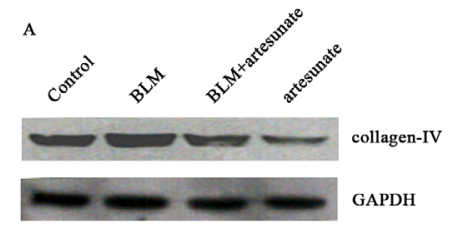

B

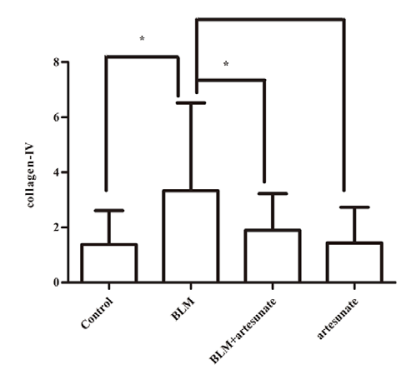

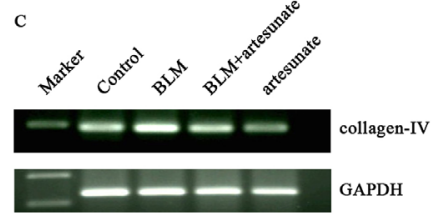

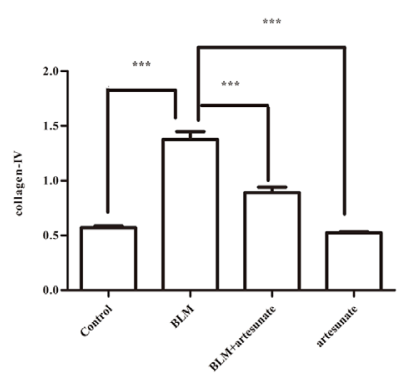

Figure 3. Expression of collagen-IV was downregulated by artesunate in lung tissues and lung fibroblasts. A. Representative western blotting results for collagen-IV protein levels in lung tissues. B. Summarized western blotting results $(\mathrm{N}=3)$. C. Representative RT-PCR results for collagen-IV mRNA levels in lung fibroblasts. D. Summarized RT-PCR results $(\mathrm{N}=3)$. Results are reported as means \pm standard deviation. $* \mathrm{P}<0.05$ and $* * * \mathrm{P}<0.001$.

\section{Artesunate upregulates MMP-2 and MMP-9 expression in BLM-induced pulmonary fibrosis in rats}

It has been reported that MMP-2 is widely expressed in fibrotic lung tissues and can degrade type IV collagen. We observed that collagen-IV was upregulated in fibrotic lung tissues and artesunate inhibited collagen-IV accumulation. We then examined MMP-2 protein levels and found that bleomycin treatment caused MMP-2 protein elevation and artesunate enhanced the effect of bleomycin on MMP-2, but artesunate alone did not have an effect on MMP-2 levels (Figure 4A and B). We also detected increased MMP-9 protein levels after bleomycin injection compared with the control group, and artesunate enhanced the effect of bleomycin (Figure 5A and B). To confirm the data from lung tissues, we performed RT-PCR to detect MMP-2 and -9 mRNA levels in cultured lung fibroblasts and obtained similar results (Figures 4C-D and 5C-D).

\section{Artesunate inhibits TIMP-1 and TIMP-2 expression in BLM-induced pulmonary fibrosis in rats}

TIMP-1 and 2 are the inhibitors of MMP-2 and 9, respectively, (Nagase et al., 2006) and their abnormal activity may lead to the chronic impaired tissue remodeling and abnormal collagen deposition. Our western blotting results demonstrated that bleomycin treatment led to increased TIMP-1 and TIMP-2 expression, and artesunate administration attenuated this effect (Figures 6A-B and 7A-B). The TIMP-1 and TIMP-2 mRNA levels in cultured fibroblasts detected by RTPCR were consistent with the western blotting results in lung tissues (Figures 6C-D and 7C-D). 


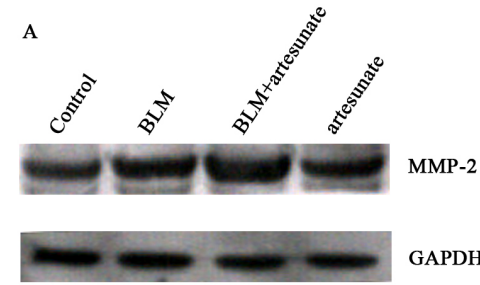

B

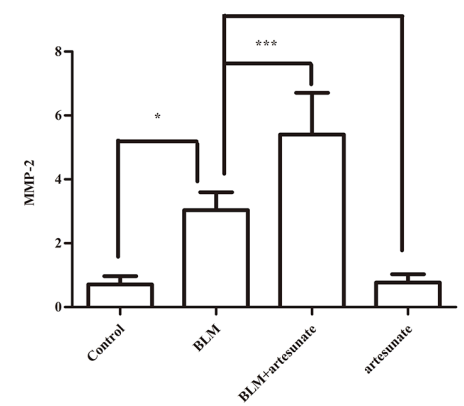

C
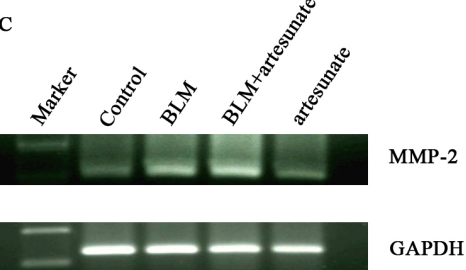

D

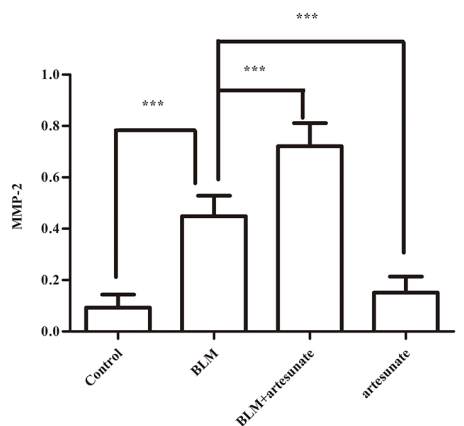

Figure 4. MMP-2 expression was upregulated by artesunate in lung tissues and lung fibroblasts. A. Representative western blotting results for MMP-2 protein levels in lung tissues. B. Summarized western blotting results $(\mathrm{N}=3)$. C. Representative RT-PCR results for MMP-2 mRNA levels in lung fibroblasts. D. Summarized RT-PCR results (N $=3$ ). Results are reported as means \pm standard deviation. $* \mathrm{P}<0.05$ and $* * * \mathrm{P}<0.001$.

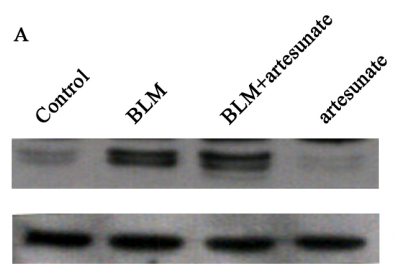

B

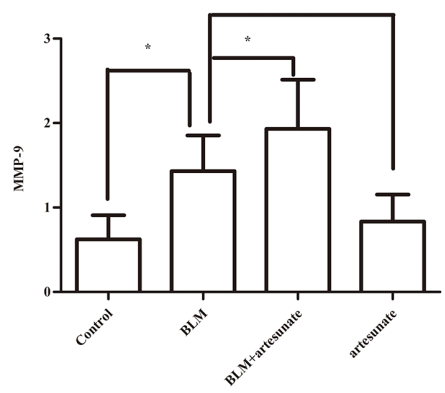

c

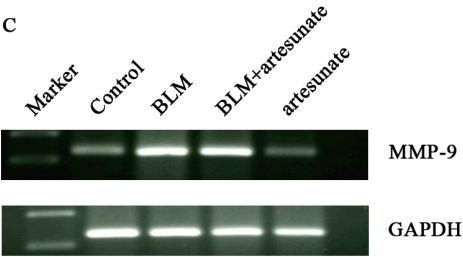

D

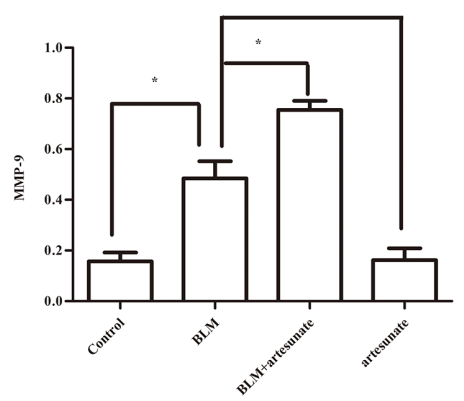

Figure 5. MMP-9 expression was upregulated by artesunate in lung tissues and lung fibroblasts. A. Representative western blotting results for MMP-9 protein levels in lung tissues. B. Summarized western blotting results $(\mathrm{N}=3)$. C. Representative RT-PCR results for MMP-9 mRNA levels in lung fibroblasts. D. Summarized RT-PCR results (N $=3$ ). Results are reported as means \pm standard deviation. $* \mathrm{P}<0.05$ and $* * * \mathrm{P}<0.001$. 


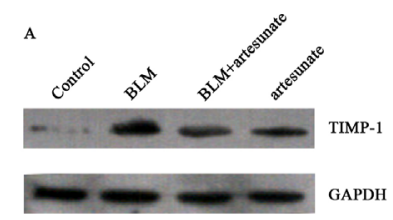

B

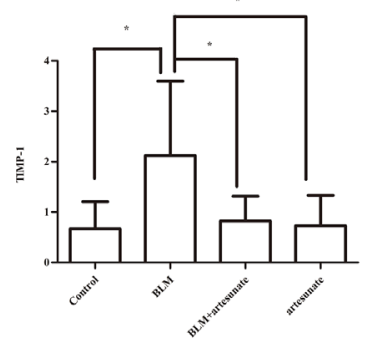

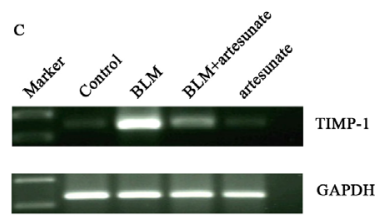

D

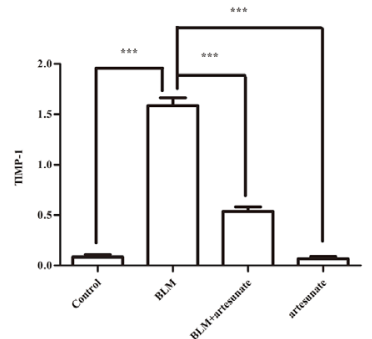

Figure 6. TIMP-1 expression was downregulated by artesunate in lung tissues and lung fibroblasts. A. Representative western blotting results for TIMP-1 protein levels in lung tissues. B. Summarized western blotting results $(\mathrm{N}=3)$. C. Representative RT-PCR results for TIMP-1 mRNA levels in lung fibroblasts. D. Summarized RT-PCR results (N $=3$ ). Results are reported as means \pm standard deviation. $* \mathrm{P}<0.05$ and $* * * \mathrm{P}<0.001$.

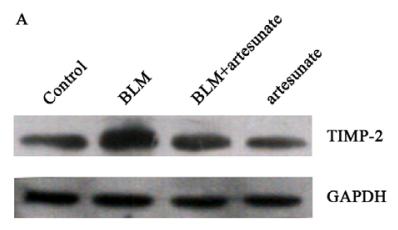

B

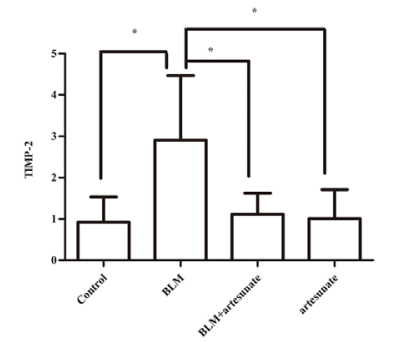

$\mathrm{C}$

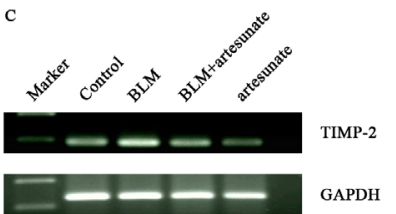

D

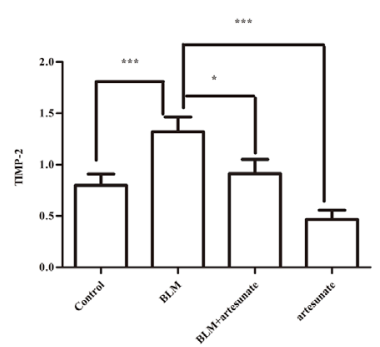

Figure 7. TIMP-2 expression was downregulated by artesunate in lung tissue and lung fibroblasts. A. Representative western blotting results for TIMP-2 protein levels in lung tissues. B. Summarized western blotting results $(\mathrm{N}=3)$. C. Representative RT-PCR results for TIMP-2 mRNA levels in lung fibroblasts. D. Summarized RT-PCR results (N $=3$ ). Results are reported as means \pm standard deviation. $* \mathrm{P}<0.05$ and $* * * \mathrm{P}<0.001$.

\section{DISCUSSION}

Pulmonary fibrosis is a chronic and progressive fibrotic lung disorder characterized by diffused alveolar inflammation and disrupted alveolar structure. Pulmonary fibrosis gradually replaces normal lung tissues, leading to severe lung dysfunction. Inflammation after epithelial injury and the excessive deposition of ECM are believed to be integral parts of the pathophysiology of pulmonary fibrosis (Pardo et al., 1992; Tetley, 1993). However, 
the pathogenesis of IPF is not yet completely understood, and there is no effective therapy currently available. Recently, we found that artesunate might be a potential drug for IPF. In the present study, we analyzed the mechanisms of how artesunate reduced bleomycin-induced alveolitis and pulmonary fibrosis in rats.

Collagen-IV is the major component of the alveoli basement membrane, and its excessive deposition can induce lung tissue remodeling and alveolar interval progressive thickening, making the alveolar cavity shrink or even disappear, eventually leading to pulmonary fibrosis. Artesunate treatment resulted in decreased collagen-IV protein in lung tissues and decreased collagen-IV mRNA in primary lung fibroblast cells, indicating that the anti-fibrotic effect of artesunate might be involve, at least partially, downregulation collagen-IV expression. MMP-2 and MMP-9 are well-known owing to their ability to degrade collagen-IV, and their increased activity leads to various pulmonary diseases including pulmonary fibrosis (Krämer et al., 2008; Bridle et al., 2009; Korfhagen et al., 2009). MMP-2 and MMP-9 play an important role in the inefficient remodeling of damaged lung tissue (Checa et al., 2008; Yamashita et al., 2011; Song et al., 2013). In the current study, we demonstrated that the protein levels of MMP-2 and MMP-9 were upregulated in lung tissues by bleomycin treatment and artesunate enhanced this effect, which was consistent with the mRNA levels in lung fibroblasts. The activity of TIMP-1 or TIMP-2 is increased in pulmonary fibrosis (Yang et al., 2007), especially in the acute phase of pulmonary fibrosis or acute lung injury model. It has also been suggested that TIMPs are related to the process of bronchial epithelial cell regeneration (O'Kane et al., 2009). We found in the present study that bleomycin induced an increase in TIMP-1 and TIMP-2 at the protein level in lung tissues and at the RNA level in lung fibroblasts, whereas artesunate treatment caused a decrease in TIMP-1 and TIMP-2 at the protein level in lung tissues and at the RNA level in lung fibroblasts.

The main clinical manifestation of IPF is dyspnea, and the primary treatment for IPF is glucocorticoid, which has no obvious effect. Our group recently found that artesunate has anti-fibrotic effects in a pulmonary fibrosis rat model, and artesunate inhibits proliferation of embryonic lung fibroblasts through the TGF-beta1/Smad pathway (Wang et al., 2015a). This suggests that the anti-fibrotic effect of artesunate may be associated with alterations in these pro-fibrotic molecules, and thus, targeting these pro-fibrotic molecules might be a promising therapy for pulmonary fibrosis. Previous reports have found that some medicines such as rapamycin have anti-fibrotic function by reducing the ratio of MMP-9 and TIMP-1 and inhibiting the degradation of ECM (Jin et al., 2014). Our current findings demonstrated that artesunate inhibits pulmonary fibrosis by regulating the expression of certain pro-fibrotic proteins, including collagen-IV, TIMP-1, and 2, as well as MMP-2 and 9. Although expression of MMP-2 and MMP-9 increased after bleomycin treatment, their activities might be inhibited by the increased expression of TIMP-1 and 2, leading to ECM accumulation and pulmonary fibrosis aggravation. After artesunate treatment, the inhibitory effects of TIMP-1 and TIMP-2 on MMP-2 and 9 decreased, resulting in improved lung fibrosis. The current study confirmed that ECM degradation is not only determined by the amount of MMPs in the tissues, but also by the ratio of MMPs to TIMPs (Anikina et al., 2013). The present study provided insight into how artesunate alleviates pulmonary fibrosis, but further investigation should be carried out to unveil the mechanisms by which artesunate affects the lung fibroblast phenotype transformation. 


\section{Conflicts of interest}

The authors declare no conflict of interest.

\section{ACKNOWLEDGMENTS}

Research supported by the Natural Science Foundation of Guangxi (grant \#2014GXNSFAA118151) and National Natural Science Foundation of China (grant \#81360010). G. Huang was supported by Hundred Talents Program of Guangxi.

\section{REFERENCES}

Akkoc A and Kahraman MM (2012). Matrix metalloproteinase expression in primary lung fibroblasts of layer type chickens. Biotech. Histochem. 87: 201-207. http://dx.doi.org/10.3109/10520295.2011.604042

Anikina AG, Potapova OV, Shkurupii VA and Shestopalov AM (2013). Role of matrix metalloproteinases and their inhibitor in the development of early pulmonary fibrosis in mice infected with influenza $\mathrm{A} / \mathrm{H} 5 \mathrm{~N} 1 \mathrm{~A} /$ goose/ Krasnoozerskoye/627/05 virus. Bull. Exp. Biol. Med. 156: 11-14. http://dx.doi.org/10.1007/s10517-013-2265-3

Bridle KR, Popa C, Morgan ML, Sobbe AL, et al. (2009). Rapamycin inhibits hepatic fibrosis in rats by attenuating multiple profibrogenic pathways. Liver Transpl. 15: 1315-1324.http://dx.doi.org/10.1002/lt.21804

Checa M, Ruiz V, Montaño M, Velázquez-Cruz R, et al. (2008). MMP-1 polymorphisms and the risk of idiopathic pulmonary fibrosis. Hum. Genet. 124: 465-472. http://dx.doi.org/10.1007/s00439-008-0571-z

Dancer RC, Wood AM and Thickett DR (2011). Metalloproteinases in idiopathic pulmonary fibrosis. Eur. Respir. J. 38: 1461-1467.http://dx.doi.org/10.1183/09031936.00024711

du Bois RM (2010). Strategies for treating idiopathic pulmonary fibrosis. Nat. Rev. Drug Discov. 9: 129-140. http://dx.doi. org $/ 10.1038 / \mathrm{nrd} 2958$

García-de-Alba C, Becerril C, Ruiz V, González Y, et al. (2010). Expression of matrix metalloproteases by fibrocytes: possible role in migration and homing. Am. J. Respir. Crit. Care Med. 182: 1144-1152. http://dx.doi.org/10.1164/ rccm.201001-00280C

Jin X, Dai H, Ding K, Xu X, et al. (2014). Rapamycin attenuates bleomycin-induced pulmonary fibrosis in rats and the expression of metalloproteinase-9 and tissue inhibitors of metalloproteinase-1 in lung tissue. Chin. Med. J. (Engl.) 127: 1304-1309.

Kiani A, Mostafaie A, Shirazi FH and Ghazanfari T (2013). Serum profiles of matrix metalloproteinases and their tissue inhibitors in long-term pulmonary complication induced by sulfur mustard: Sardasht-Iran Cohort Study (SICS). Int. Immunopharmacol. 17: 964-967. http://dx.doi.org/10.1016/j.intimp.2012.12.025

Kisarova YA and Korolenko TA (2012). Tissue inhibitors of matrix metalloproteinases 1 and 2 and matrix metalloproteinase activity in the serum and lungs of mice with lewis lung carcinoma. Bull. Exp. Biol. Med. 153: 874-877. http://dx.doi. org/10.1007/s10517-012-1848-8

Korfhagen TR, Le Cras TD, Davidson CR, Schmidt SM, et al. (2009). Rapamycin prevents transforming growth factor-alphainduced pulmonary fibrosis. Am. J. Respir. Cell Mol. Biol. 41: 562-572. http://dx.doi.org/10.1165/rcmb.2008-0377OC

Krämer S, Wang-Rosenke Y, Scholl V, Binder E, et al. (2008). Low-dose mTOR inhibition by rapamycin attenuates progression in anti-thy1-induced chronic glomerulosclerosis of the rat. Am. J. Physiol. Renal Physiol. 294: F440-F449. http://dx.doi.org/10.1152/ajprenal.00379.2007

Leppäranta O, Sens C, Salmenkivi K, Kinnula VL, et al. (2012). Regulation of TGF- $\beta$ storage and activation in the human idiopathic pulmonary fibrosis lung. Cell Tissue Res. 348: 491-503.http://dx.doi.org/10.1007/s00441-012-1385-9

Nagase H, Visse R and Murphy G (2006). Structure and function of matrix metalloproteinases and TIMPs. Cardiovasc. Res. 69: 562-573.http://dx.doi.org/10.1016/j.cardiores.2005.12.002

Li HX, Liu H, Wang CM, Wang HJ, et al. (2014). Artesunate restraining MAPK passage by smad7 to resist pulmonary fibrosis. Eur. Rev. Med. Pharmacol. Sci. 18: 3199-3204.

O'Kane CM, McKeown SW, Perkins GD, Bassford CR, et al. (2009). Salbutamol up-regulates matrix metalloproteinase-9 in the alveolar space in the acute respiratory distress syndrome. Crit. Care Med. 37: 2242-2249. http://dx.doi. org/10.1097/CCM.0b013e3181a5506c

Pardo A and Selman M (2012). Role of matrix metaloproteases in idiopathic pulmonary fibrosis. Fibrogenesis Tissue Repair 5 (Suppl 1): S9. 
Pardo A, Selman M, Ramírez R, Ramos C, et al. (1992). Production of collagenase and tissue inhibitor of metalloproteinases by fibroblasts derived from normal and fibrotic human lungs. Chest 102: 1085-1089. http://dx.doi.org/10.1378/ chest.102.4.1085

Song JW, Do KH, Jang SJ, Colby TV, et al. (2013). Blood biomarkers MMP-7 and SP-A: predictors of outcome in idiopathic pulmonary fibrosis. Chest 143: 1422-1429. http://dx.doi.org/10.1378/chest.11-2735

Szapiel SV, Elson NA, Fulmer JD, Hunninghake GW, et al. (1979). Bleomycin-induced interstitial pulmonary disease in the nude, athymic mouse. Am. Rev. Respir. Dis. 120: 893-899.

Tetley TD (1993). New perspectives on basic mechanisms in lung disease. 6. Proteinase imbalance: its role in lung disease. Thorax 48: 560-565. http://dx.doi.org/10.1136/thx.48.5.560

Van Doren SR (2015). Matrix metalloproteinase interactions with collagen and elastin. Matrix Biol. 44-46: 224-231.http:// dx.doi.org/10.1016/j.matbio.2015.01.005

Wang C, Xuan X, Yao W, Huang G, et al. (2015a). Anti-profibrotic effects of artesunate on bleomycin-induced pulmonary fibrosis in Sprague Dawley rats. Mol. Med. Rep. 12: 1291-1297.

Wang C, Li Z, Liu X, Peng Q, et al. (2015b). Effect of Liuweibuqi capsule, a Chinese patent medicine, on the JAK1/STAT3 pathway and MMP9/TIMP1 in a chronic obstructive pulmonary disease rat model. J. Tradit. Chin. Med.35: 54-62.

Yamashita CM, Dolgonos L, Zemans RL, Young SK, et al. (2011). Matrix metalloproteinase 3 is a mediator of pulmonary fibrosis. Am. J. Pathol. 179: 1733-1745. http://dx.doi.org/10.1016/j.ajpath.2011.06.041

Yang K, Palm J, König J, Seeland U, et al. (2007). Matrix-Metallo-Proteinases and their tissue inhibitors in radiationinduced lung injury. Int. J. Radiat. Biol. 83: 665-676. http://dx.doi.org/10.1080/09553000701558977 